\title{
ARHGAP6 regulates the proliferation, migration and invasion of lung cancer cells
}

\author{
YONGKAI WU, MING XU, RONG HE, KE XU and YEGANG MA \\ Department of Thoracic Surgery, Liaoning Cancer Hospital and Institute, Shenyang 110042, P.R. China
}

Received April 9, 2018; Accepted September 17, 2018

DOI: $10.3892 /$ or.2019.7031

\begin{abstract}
Lung cancer, a leading cause of cancer-related deaths, is frequently diagnosed in both males and females worldwide. In the present study, the Ras homologue GTPase activation protein 6 (ARHGAP6), which belongs to the Rho GTPase-activating protein (RhoGAP) family, was found to have low expression in tumor tissues from patients with lung cancer, accompanied by high expression of matrix metalloproteinase-9 (MMP9) and vascular endothelial growth factor (VEGF). In A549 and H1299 cells, upregulation of ARHGAP6 inhibited tumor growth and metastasis and reduced the levels of MMP9, VEGF and p-STAT3, while the levels STAT3 were unchanged, as demonstrated by CCK-8, migration and invasion assays as well as western blot analysis. In addition, interleukin 6 (IL-6)-induced migration, invasion and MMP9 and VEGF expression, and STAT3 signaling activity were suppressed by ARHGAP6 upregulation. Based on these data, we concluded that ARHGAP6 is critically important in lung cancer progression and that upregulation of ARHGAP6 benefits the treatment and prevention of lung cancer, possibly through the suppression of MMP9, VEGF and STAT3 signaling activation.
\end{abstract}

\section{Introduction}

Lung cancer, which is frequently diagnosed in both males and females, is the leading cause of cancer-related deaths worldwide $(1,2)$. In 2008 , there were more than 1.6 million lung cancer cases diagnosed, comprising $\sim 12.7 \%$ of all new cancer cases (3). In general, small cell lung cancer (SCLC) and non-small cell lung cancer (NSCLC) are the two major types of lung cancer. Evidence suggests that tobacco smoking is the main cause of lung cancer, accounting for probably $90 \%$ of all lung cancer diagnoses $(4,5)$. The risk of lung cancer

Correspondence to: Dr Yegang Ma, Department of Thoracic Surgery, Liaoning Cancer Hospital and Institute, 44 Xiaoheyan Road, Shenyang 110042, P.R. China

E-mail: myggsmyc@163.com

Key words: ARHGAP6, lung cancer, MMP9, VEGF, STAT3 signaling, IL-6 among active smokers is 10 -fold higher than that among non-smokers (6). Despite improved survival intervals, the survival rate for lung cancer is still discouragingly low (7).

Migration and invasion are widely recognized as two major hallmarks of malignancy and are closely related to the biological behavior of cancer cells. Actin, a main component of the cytoskeleton, plays an important role in cell movement $(8,9)$. The Ras homologue GTPase activation protein 6 (ARHGAP6) is a novel Rho GTPase-activating protein (RhoGAP) gene that plays a crucial role in regulating actin polymerization in several cellular processes, including tumor growth and metastasis $(10,11)$. It was reported that ARHGAP6 inhibited the proliferation and metastasis of cervical carcinoma, caused cell cycle arrest, and induced cell apoptosis (12). ARHGAP6 isoform 1 variant, an $\mathrm{Hb} 3$ antigen, may be a novel biomarker of the progression of colorectal cancer (CRC); $8.3 \%$ of patients with CRC develop pulmonary metastases despite undergoing curative surgery (13). However, the role of ARHGAP6 expression in lung cancer remains unclear. Matrix metalloproteinase-9 (MMP9) and vascular endothelial growth factor (VEGF) are commonly highly expressed in lung cancer and play an important role in lung cancer progression (14-16). Studies have associated MMP9 expression to tumor growth, metastasis and angiogenesis $(17,18)$. VEGF is a major growth factor involved in angiogenesis, and VEGF-mediated angiogenesis is a crucial process for tumor growth and metastasis $(19,20)$. In addition, signal transducer and activator of transcription 3 (STAT3), a transcription factor, has been found to be activated in lung cancer (21). Indirectly or directly inhibiting STAT3 activity affects the transcription of several genes, including VEGF, somewhat inhibits angiogenesis and affects the formation of tumors $(22,23)$. A previous study reported that interleukin 6 (IL-6) acted as a strong activator of STAT3 signaling in lung cancer (24). IL-6 is a key cytokine frequently upregulated in cancer and considered a mediator of malnutrition in lung cancer patients $(25,26)$.

In this study, significantly reduced ARHGAP6 levels were observed in tumor tissues from patients with lung cancer, accompanied by high levels of MMP9 and VEGF, which indicated that ARHGAP6 was involved in lung cancer progression. In vitro upregulation of ARHGAP6 significantly inhibited the growth and metastasis of A549 and H1299 cells, accompanied by decreased protein levels of MMP9, VEGF and p-STAT3. Moreover, IL-6-induced migration, invasion and expression of MMP9, VEGF and p-STAT3 were suppressed by ARHGAP6 
upregulation. These results indicated that ARHGAP6 upregulation may benefit lung cancer patients through the suppression of MMP9, VEGF and STAT3 signaling.

\section{Materials and methods}

Lung cancer tissue and adjacent normal tissue. After written informed consent was obtained, twenty-five pairs of tumor and adjacent normal tissue from lung cancer patients (13 men and 12 women among the age of 50-80 years) treated at Liaoning Cancer Hospital and Institute (Liaoning, China) were collected from May to December 2016 and immediately frozen in liquid nitrogen. After pretreatment, the expression of ARHGAP6, MMP9 and VEGF in these samples was detected by real-time PCR. All experiments in this study were approved by the Ethics Committee of Liaoning Cancer Hospital and Institute.

Cell culture. The A549 and H1299 human lung cancer cell lines and the 16HBE pulmonary epithelial cell line were purchased from the Cell Bank of the Chinese Academy of Sciences (Shanghai, China). These cells were cultured in RPMI-1640 medium (product no. SH30809.01B; HyClone Laboratories; GE Healthcare Life Sciences, Logan, UT, USA) containing $10 \%$ fetal bovine serum (cat. no. 16000-044; Gibco; Thermo Fisher Scientific, Inc., Waltham, MA, USA) and $1 \%$ antibiotics (100X, a mixture of penicillin and streptomycin; product code: P1400-100; Beijing Solarbio Science \& Technology Co., Ltd., Beijing, China) in a 5\% $\mathrm{CO}_{2}$ incubator (Thermo Forma 3111; Thermo Fisher Scientific, Inc.) at $37^{\circ} \mathrm{C}$. The medium was replaced according to the growth demands of the cells during incubation.

Construction of the lentivirus. The mRNA sequence of the target gene was queried in NCBI, and primers and restriction sites were then designed for the coding sequence (CDS) and the selected vector. The 2925-bp full-length CDS of ARHGAP6 (NM_013427.2) synthesized by Genewiz Company (Shanghai, China) was inserted into the EcoRI/BamHI restriction sites of the pLVX-Puro plasmid, and the resulting plasmid was confirmed by DNA sequencing (Majorbio Bio-Pharm Technology Co., Ltd., Shanghai, China). The core plasmid pLVX-Puro-ARHGAP6 and the viral packaging plasmids psPAX2 and pMD2G (Addgene, Inc., Cambridge, MA, USA) were co-transfected into 293T cells with Lipofectamine 2000 (Invitrogen; Thermo Fisher Scientific, Inc.). After $48 \mathrm{~h}$ of transfection, the virus particles in the medium were collected (15).

Experimental groups. To upregulate the expression of ARHGAP6 in the A549 and H1299 cell lines, lentivirus-mediated RNA overexpression was used. A549 and H1299 cells were divided into three groups: Infected with medium (control), infected with negative control lentivirus (vector) and infected with ARHGAP6 recombinant lentivirus (oe-ARHGAP6). After $48 \mathrm{~h}$ of infection, real-time PCR, western blot analysis and Transwell assays were carried out. In addition, proliferation assays were performed at $0,24,48$ and $72 \mathrm{~h}$.

To further investigate the effects of ARHGAP6 on lung cancer cells, A549 and H1299 cells were divided into three groups and then treated with medium (control), negative control lentivirus and $50 \mathrm{ng} / \mathrm{ml} \mathrm{IL-6}$ (Vector+IL-6), or ARHGAP6 recombinant lentivirus and $50 \mathrm{ng} / \mathrm{ml} \mathrm{IL-6}$ (oe-ARHGAP6+IL-6). After $48 \mathrm{~h}$ of treatment, western blot analysis and Transwell assays were carried out.

Proliferation assay. A549 and H1299 cells in the logarithmic growth phase were digested with $0.25 \%$ trypsin (Beijing Solarbio Science \& Technology Co., Ltd.), inoculated in triplicate in 96 -well culture plates at a density of $3 \times 10^{3}$ cells/well, and cultured in a humidified incubator overnight at $37^{\circ} \mathrm{C}$ with 5\% $\mathrm{CO}_{2}$. The following day, the cells were infected with medium, negative control lentivirus, or ARHGAP6 recombinant lentivirus, and after $0,24,48$ and $72 \mathrm{~h}$ of infection, $100 \mu \mathrm{l}$ of $10 \%$ Cell Counting Kit- 8 (CCK-8; SAB, College Park, MD, USA) solution in serum-free medium was added to each well, and the plates were then placed in an incubator for $1 \mathrm{~h}$. The absorbance at $450 \mathrm{~nm}$ was assessed by a microplate reader (Perlong Medical Equipment Co., Ltd., Beijing, China).

Real-time polymerase chain reaction (RT-PCR) assay. Total RNA was isolated from lentivirus-infected A549 and H1299 cells by TRIzol (Invitrogen; Thermo Fisher Scientific, Inc.), and RNA quality was confirmed by $1 \%$ agarose gel electrophoresis after quantification. A reverse transcriptase kit (Fermentas; Thermo Fisher Scientific, Inc.) was used to reverse transcribe RNA into cDNA. RT-PCR with cDNA as a template was conducted on an ABI PRISM 7300 instrument (Applied Biosystems; Thermo Fisher Scientific, Inc.) using a SYBR-Green PCR kit (Thermo Fisher Scientific, Inc.). The data were normalized to GAPDH, and the mRNA level of ARHGAP6 was calculated by the $2^{-\Delta \Delta C q}$ method (27). The primers were as follows: ARHGAP6, 5'-GAA TTTGACCGTGGGATTG-3' and 5'-CAGGGAGGTAGAAGG TATATG-3'; GAPDH, 5'-AATCCCATCACCATCTTC-3' and 5'-AGGCTGTTGTCATACTTC-3'. The RT-PCR procedure was as follows: $95^{\circ} \mathrm{C}$ for $10 \mathrm{~min}$, followed by 40 cycles at $95^{\circ} \mathrm{C}$ for $15 \mathrm{sec}$ and $60^{\circ} \mathrm{C}$ for $45 \mathrm{sec}$; one cycle at $95^{\circ} \mathrm{C}$ for $15 \mathrm{sec}$ and $60^{\circ} \mathrm{C}$ for $1 \mathrm{~min}$; and one cycle at $95^{\circ} \mathrm{C}$ for $15 \mathrm{sec}$ and $60^{\circ} \mathrm{C}$ for $15 \mathrm{sec}(28)$.

Western blot analysis. Treated A549 and H1299 cells were lysed in RIPA buffer (Beijing Solarbio Science \& Technology Co., Ltd.) containing protease and phosphatase inhibitors and incubated for $\sim 30 \mathrm{~min}$ on ice. The proteins in the supernatant of the cell lysates were collected after centrifugation for $10 \mathrm{~min}$ at $12,000 \mathrm{x} \mathrm{g}$ at $4^{\circ} \mathrm{C}$ and quantified by a BCA quantification kit (Thermo Fisher Scientific, Inc.). After being separated by SDS-PAGE (JRDUN Biotechnology Co., Ltd, Shanghai, China), the proteins on the gel were transferred onto polyvinylidene fluoride (PVDF) membranes (EMD Millipore, Billerica, MA, USA) by semidry electroblotting. The PVDF membranes were blocked in 5\% skimmed milk (BD Biosciences, Franklin Lakes, NJ, USA) for $1 \mathrm{~h}$ at room temperature, followed by incubation with primary antibodies against ARHGAP6 (1:1,000; cat. no. NBPI-80837; Biocompare, San Francisco, CA, USA), MMP9 (1:1,000; cat. no. ab38898), VEGF (1:2,000; cat. no. ab69479), STAT3 (1:100; cat. no. ab50761), p-STAT3 (1:20,0000; cat. no. ab76315; all from Abcam, Cambridge, MA, USA) or GAPDH (1:2,000; cat. no. 5174; Cell Signaling Technology, Inc., Danvers, MA, USA) at $4^{\circ} \mathrm{C}$ overnight with gentle shaking. After 5-6 washes with TBST, the membranes were incubated with secondary antibodies 

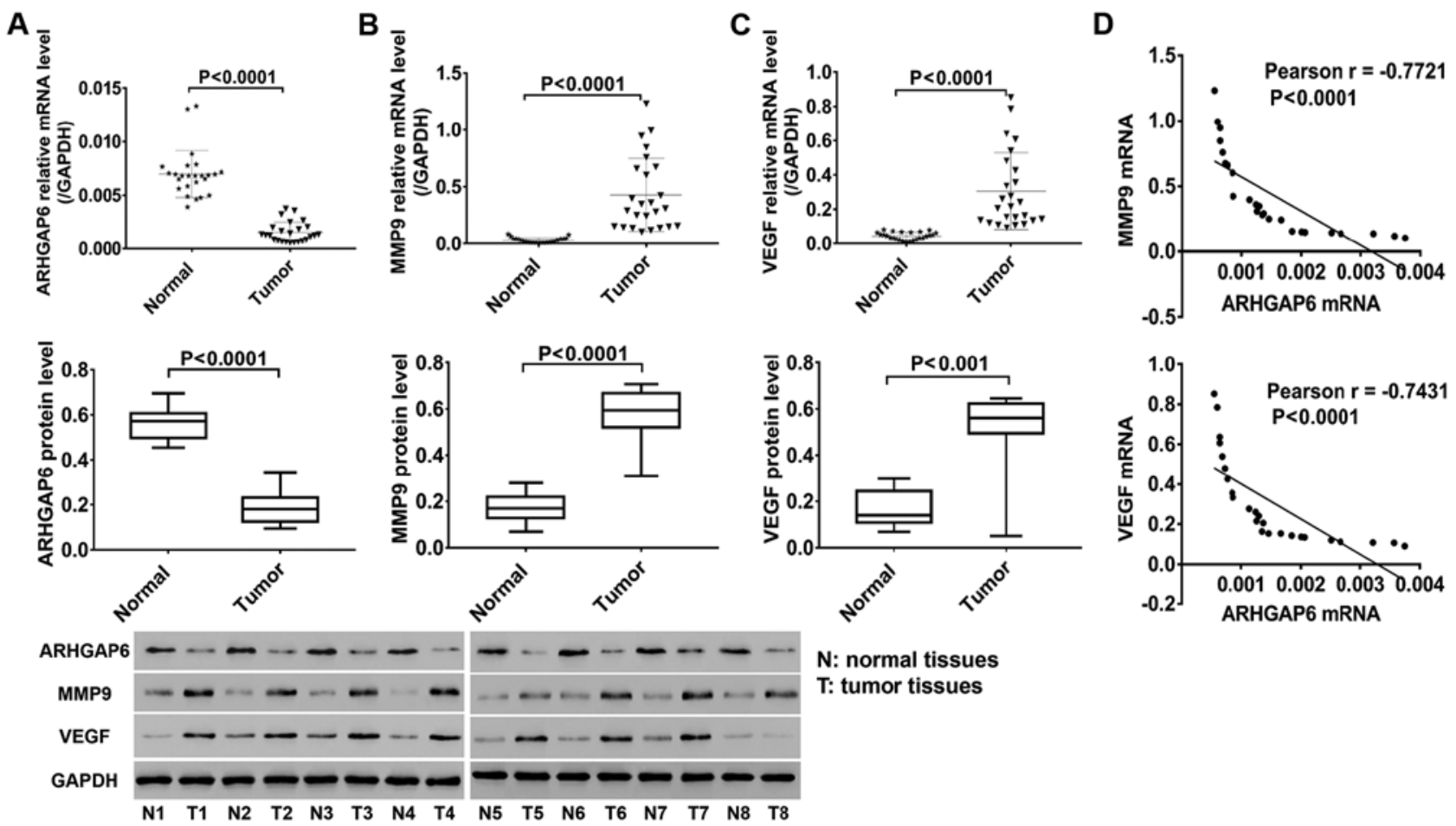

Figure 1. ARHGAP6 levels are significantly decreased in tumor tissues from lung cancer patients. Twenty-five pairs of tumor and adjacent normal tissues were collected from lung cancer patients. After RNA and protein extraction, the expression of (A) ARHGAP6, (B) MMP9, and (C) VEGF in these tissues was detected by RT-PCR and western blotting. (D) Pearson analysis was applied to determine correlations between ARHGAP6 and MMP9, and ARHGAP6 and VEGF (P<0.0001). ARHGAP6, Ras homologue GTPase activation protein 6; MMP9, matrix metalloproteinase-9; VEGF, vascular endothelial growth factor.

(1:1,000; Beyotime Institute of Biotechnology, Haimen, China) of goat anti-rabbit (cat. no. A0208) and goat anti-mouse (cat.no. A0216) for $1 \mathrm{~h}$ at $37^{\circ} \mathrm{C}$. Finally, the target protein bands were visualized on an ECL imaging system (Tanon-5200; Tanon Science and Technology Co., Ltd., Shanghai, China) after a 5-min incubation with chemiluminescent detection reagent (EMD Millipore) and the protein levels were calculated by ImageJ software $1.47 \mathrm{v}$ (National Institutes of Health, Bethesda, MD, USA).

Cell migration and invasion assays. The migration and invasion activities of A549 and H1299 cells were measured by Transwell assays using a modified Boyden chamber (Transwell Costar; cat. no. 342; Corning Inc., Corning, NY, USA) as previously described (29). The 24-well plates and Transwell chambers were soaked in $1 \mathrm{X}$ PBS for 5 min before inoculation (one more step for the invasion assay: $80 \mu \mathrm{l}$ of Matrigel was placed in small chambers and clotted in a $37^{\circ} \mathrm{C}$ incubator for $30 \mathrm{~min}$ ). After $24 \mathrm{~h}$ of serum starvation, infected and treated cells were digested by trypsin, washed, resuspended in serum-free medium, and inoculated in the upper chamber at a density of $5 \times 10^{4}$ cells/well. Then, $0.7 \mathrm{ml}$ of RPMI-1640 medium containing $10 \%$ FBS as a chemoattractant was added to the lower chamber. After $24 \mathrm{~h}$ of incubation in a $37^{\circ} \mathrm{C}$ incubator, the migrating and invading cells on the lower side of the membrane were fixed with $1 \mathrm{ml}$ of $4 \%$ formaldehyde for $10 \mathrm{~min}$. After removing the fixative and washing once with $1 \mathrm{X}$ PBS, the cells were incubated in $1 \mathrm{ml}$ of $0.5 \%$ crystal violet for $30 \mathrm{~min}$, washed once with $1 \mathrm{X}$ PBS and dried. The number of cells that migrated and invaded from the upper chamber to the lower surface was counted with a microscope at $\mathrm{x} 200$.
Statistical analysis. GraphPad Prism 7.0 (GraphPad Software, Inc., La Jolla, CA, USA) was used to perform statistical analyses. Quantitative data comparisons between two groups were analyzed by paired Student's t-test (for parametric data), while differences among multiple groups were analyzed by one-way analysis of variance (ANOVA) followed by Tukey's multiple comparison. Pearson analysis was used to determine correlations between two groups. The data are presented as the mean \pm SD of three independent experiments, and a P-value of $<0.05$ was considered to indicate a statistically significant difference.

\section{Results}

ARHGAP6 levels are significantly decreased in tumor tissues from lung cancer patients. Twenty-five pairs of tumor and adjacent normal tissues from lung cancer patients were collected, and the expression of ARHGAP6, MMP9 and VEGF in these tissues was quantified by RT-PCR and western blotting. As shown in Fig. 1, ARHGAP6 mRNA (Fig. 1A, upper image) and protein (Fig. 1A, lower image) levels were significantly reduced in tumor tissues from lung cancer patients compared to adjacent normal tissues, accompanied by significantly increased levels of MMP9 (Fig. 1B) and VEGF (Fig. 1C). Pearson analysis revealed negative correlations between ARHGAP6 and MMP9 (Fig. 1D, upper image) and between ARHGAP6 and VEGF (Fig. 1D, lower image). These data indicated that ARHGAP6 may be implicated in the progression of lung cancer.

The expression of ARHGAP6 in the $16 H B E, A 549$ and H1299 cell lines. The expression of ARHGAP6 in three cell lines 

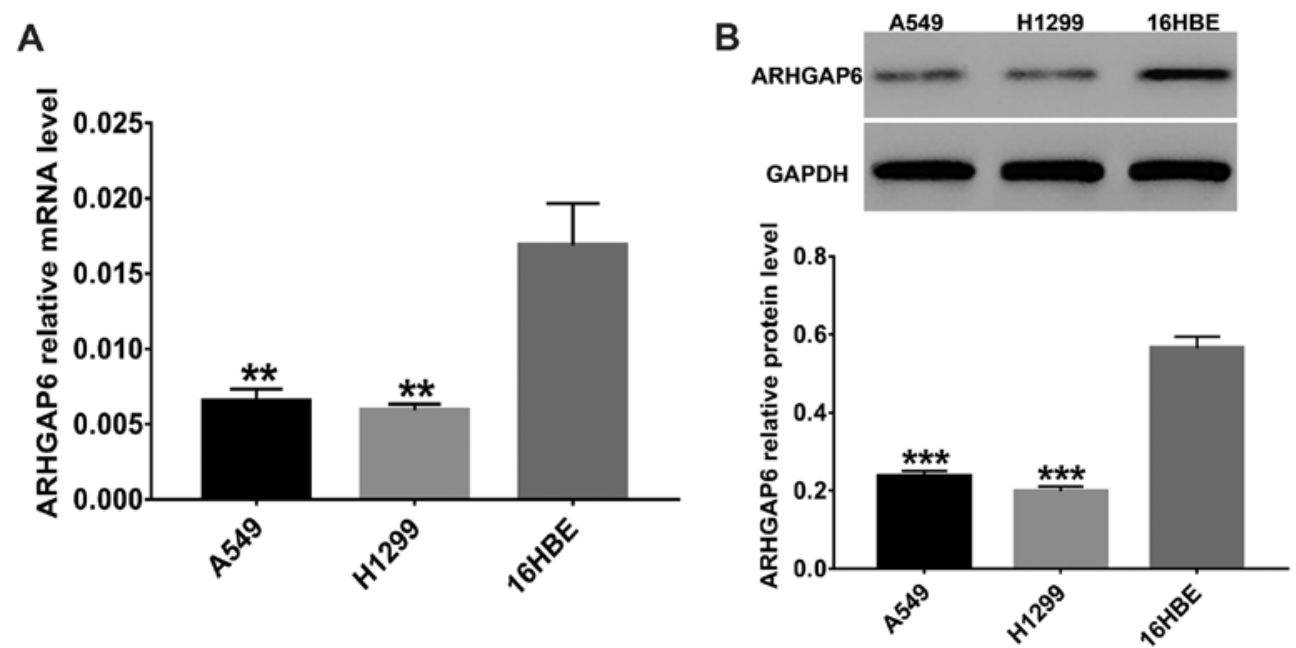

Figure 2. The expression of ARHGAP6 in the 16HBE, A549 and H1299 cell lines. In 16HBE pulmonary epithelial cells and A549 and H1299 lung cancer cell lines, the levels of (A) ARHGAP6 mRNA and (B) protein were detected by RT-PCR and western blot analysis, respectively. All data are presented as the mean \pm SD of three independent experiments $\left({ }^{* *} \mathrm{P}<0.01,{ }^{* * *} \mathrm{P}<0.001\right.$ compared to $16 \mathrm{HBE}$ cells). ARHGAP6, Ras homologue GTPase activation protein 6 .

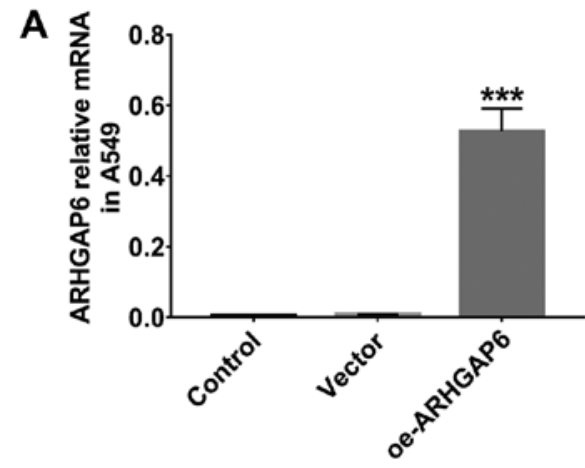

B

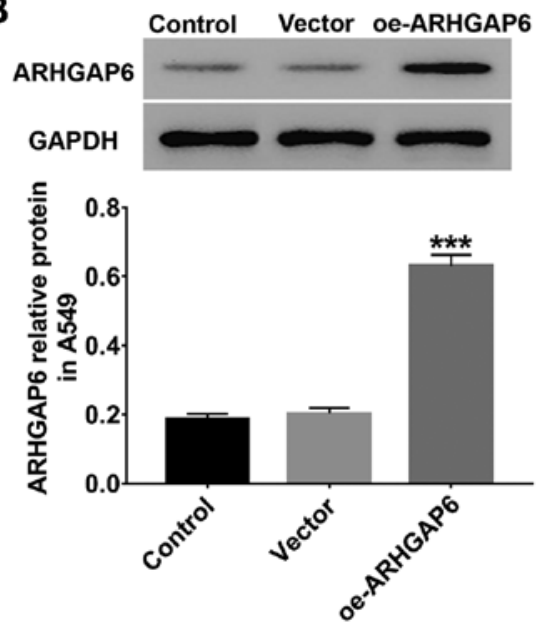

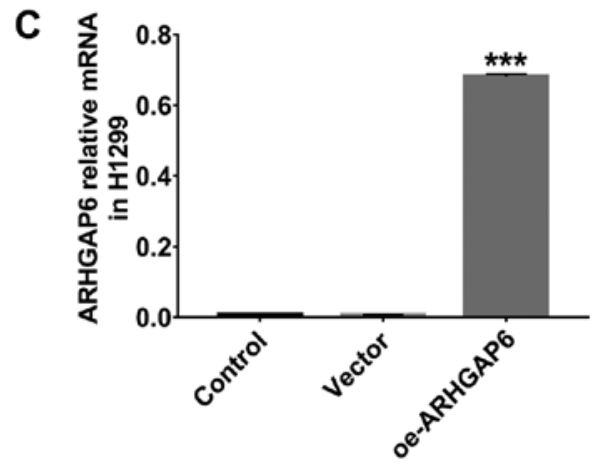

D
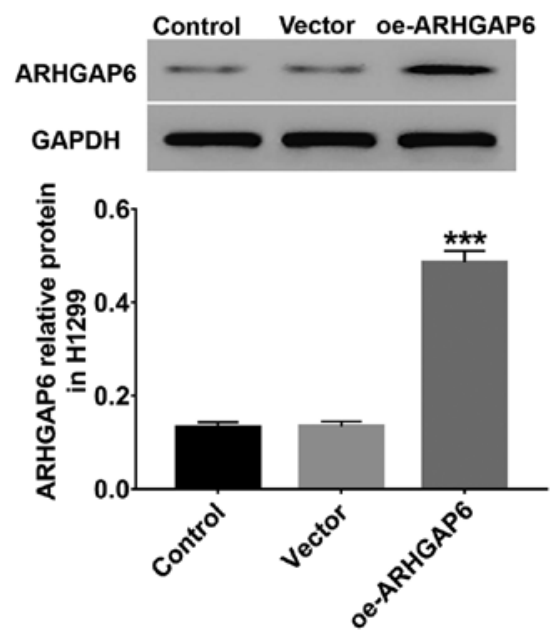

Figure 3. Upregulation of ARHGAP6 in the A549 and H1299 cell lines. (A and B) A549 and (C and D) H1299 cells were infected with ARHGAP6 recombinant lentivirus to upregulate ARHGAP6 expression. After $48 \mathrm{~h}$ of infection, the levels of ARHGAP6 mRNA and protein were quantified by RT-PCR and western blot analysis, respectively. Data are presented as the mean $\pm \mathrm{SD}$ of three independent experiments $\left({ }^{* * *} \mathrm{P}<0.001\right.$ compared to the vector). ARHGAP6, Ras homologue GTPase activation protein 6.

(16HBE, A549 and H1299) was detected in vitro. As shown in Fig. 2, the levels of ARHGAP6 mRNA (Fig. 2A) and protein (Fig. 2B) in A549 and H1299 cells were much lower than those in the 16HBE cells, which indicated that ARHGAP6 expression was closely associated with lung cancer. The A549 and H1299 cell lines were used for further study.
Upregulation of ARHGAP6 in the A549 and $H 1299$ cell lines. For further study, ARHGAP6 recombinant lentivirus was used to upregulate the expression of ARHGAP6 in A549 and H1299 cells. After $48 \mathrm{~h}$ of infection, the levels of ARHGAP6 mRNA and protein were quantified by RT-PCR and western blotting, respectively. The results revealed in Fig. 3 indicated 
A

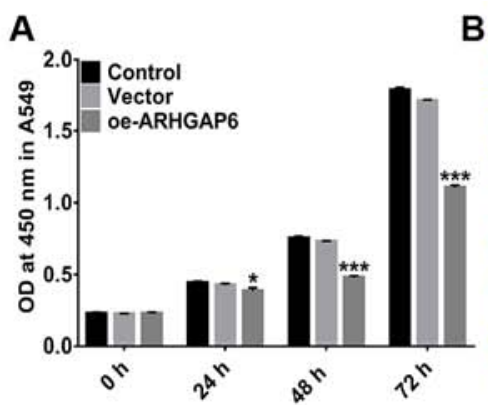

D

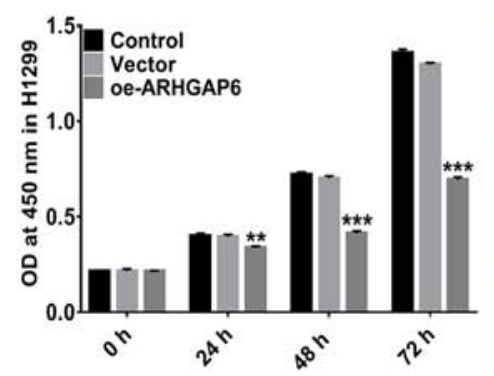

B

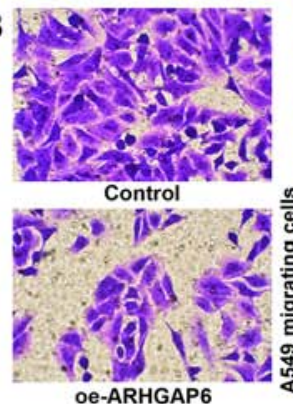

oe-ARHGAP6

E

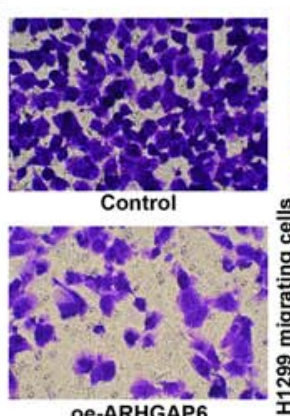

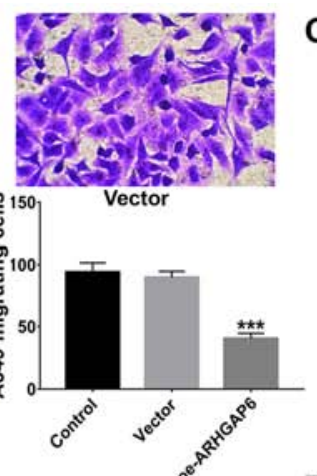

$F$

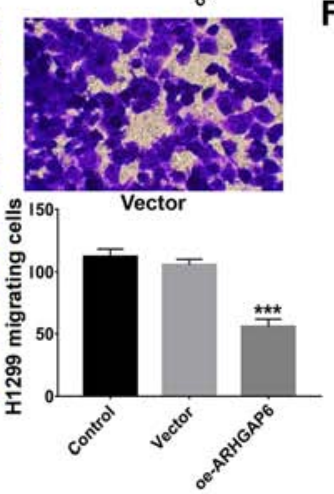

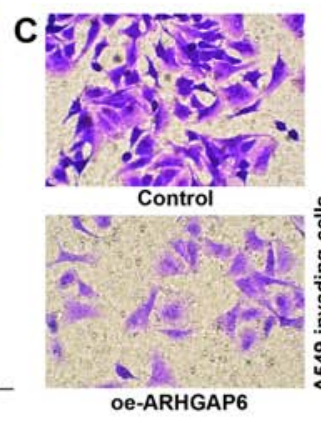
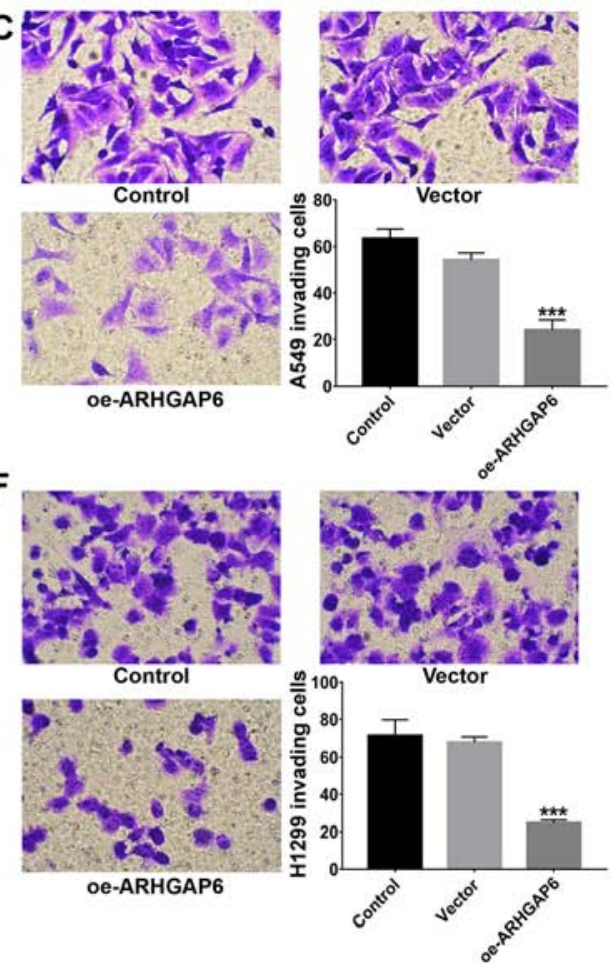

Figure 4. Effects of ARHGAP6 upregulation on the proliferation, migration and invasion of A549 and H1299 cells. After upregulating ARHGAP6 in A549 and H1299 cells, (A and D) cell proliferation was evaluated by CCK-8 assays, while the numbers of (B and E) migrating and (C and F) invading cells were determined by Transwell assays. Data are presented as the mean $\pm \mathrm{SD}$ of three independent experiments $\left({ }^{*} \mathrm{P}<0.05\right.$, ${ }^{* *} \mathrm{P}<0.01$, and ${ }^{* * *} \mathrm{P}<0.001$ compared to the vector). ARHGAP6, Ras homologue GTPase activation protein 6.

that in both A549 (Fig. 3A and B) and H1299 (Fig. 3C and D) cells, the expression of ARHGAP6 was markedly upregulated by infection with ARHGAP6 recombinant lentivirus. Therefore, the ARHGAP6 lentivirus was used in the following experiments due to its effective upregulation of ARHGAP6 expression.

Effect of ARHGAP6 upregulation on the proliferation, migration and invasion of A549 and H1299 cells. Following the upregulation of ARHGAP6 expression, the proliferation of A549 and H1299 cells was evaluated by CCK-8 assays. As revealed in Fig. 4, in both A549 (Fig. 4A) and H1299 cells (Fig. 4D), proliferation was significantly inhibited when ARHGAP6 was upregulated. In addition, the migration activities of A549 (Fig. 4B) and H1299 cells (Fig. 4E) were significantly suppressed after ARHGAP6 upregulation. Similarly, ARHGAP6-overexpressing A549 (Fig. 4C) and H1299 (Fig. 4F) cells were less invasive. These findings demonstrated that ARHGAP6 upregulation had an inhibitory effect on the growth and metastasis of lung cancer cells. Targeting ARHGAP6 may be a potential strategy for the treatment and prevention of lung cancer.

The altered expression of several associated genes after ARHGAP6 upregulation. Several cancer-associated genes (MMP9, VEGF and STAT3/p-STAT3) were analyzed to further study the effects of ARHGAP6 upregulation on lung cancer cells. After ARHGAP6 was upregulated in A549 and H1299 cells, the levels of these genes were analyzed by western blotting. As revealed in Fig. 5, in both A549 (Fig. 5A) and H1299 (Fig. 5B) cells, the protein levels of MMP9, VEGF, and p-STAT3 were markedly reduced after ARHGAP6 upregulation, while the levels of STAT3 were unchanged. Activation of MMP9 and VEGF transcription has been revealed to enhance tumor migration and the invasion of lung cancer $(30,31)$. STAT3, which mediates the transcription of several genes, including VEGF, is critically important for the progression of human lung cancer (32). Thus, we concluded that ARHGAP6 upregulation may inhibit the growth and metastasis of lung cancer cells by inhibiting MMP9, VEGF, and STAT3 signaling.

ARHGAP6 upregulation significantly suppresses IL-6-induced migration, invasion, and MMP9, VEGF and p-STAT3 expression. It has been reported that IL-6 is an important multifunctional cytokine that regulates the growth of a variety of tumors and plays a crucial role in carcinogenesis $(16,33,34)$. Here, after lentivirus infection and treatment with $50 \mathrm{ng} / \mathrm{ml}$ IL-6, the migration and invasion activities of H1299 cells and the protein levels of MMP9, VEGF, STAT3 and p-STAT3 were detected. As shown in Fig. 6, IL-6 induced the migration (Fig. 6A) and invasion (Fig. 6B) of H1299 cells, and ARHGAP6 upregulation significantly inhibited these changes induced by IL-6. Moreover, the IL-6-induced expression of MMP9, VEGF and p-STAT3 was significantly reduced by ARHGAP6 upregulation, while the level of STAT3 was unchanged (Fig. 6C). All the data demonstrated that the upregulation of ARHGAP6 inhibited lung cancer metastasis through the suppression of MMP9, VEGF, and STAT3 signaling. 

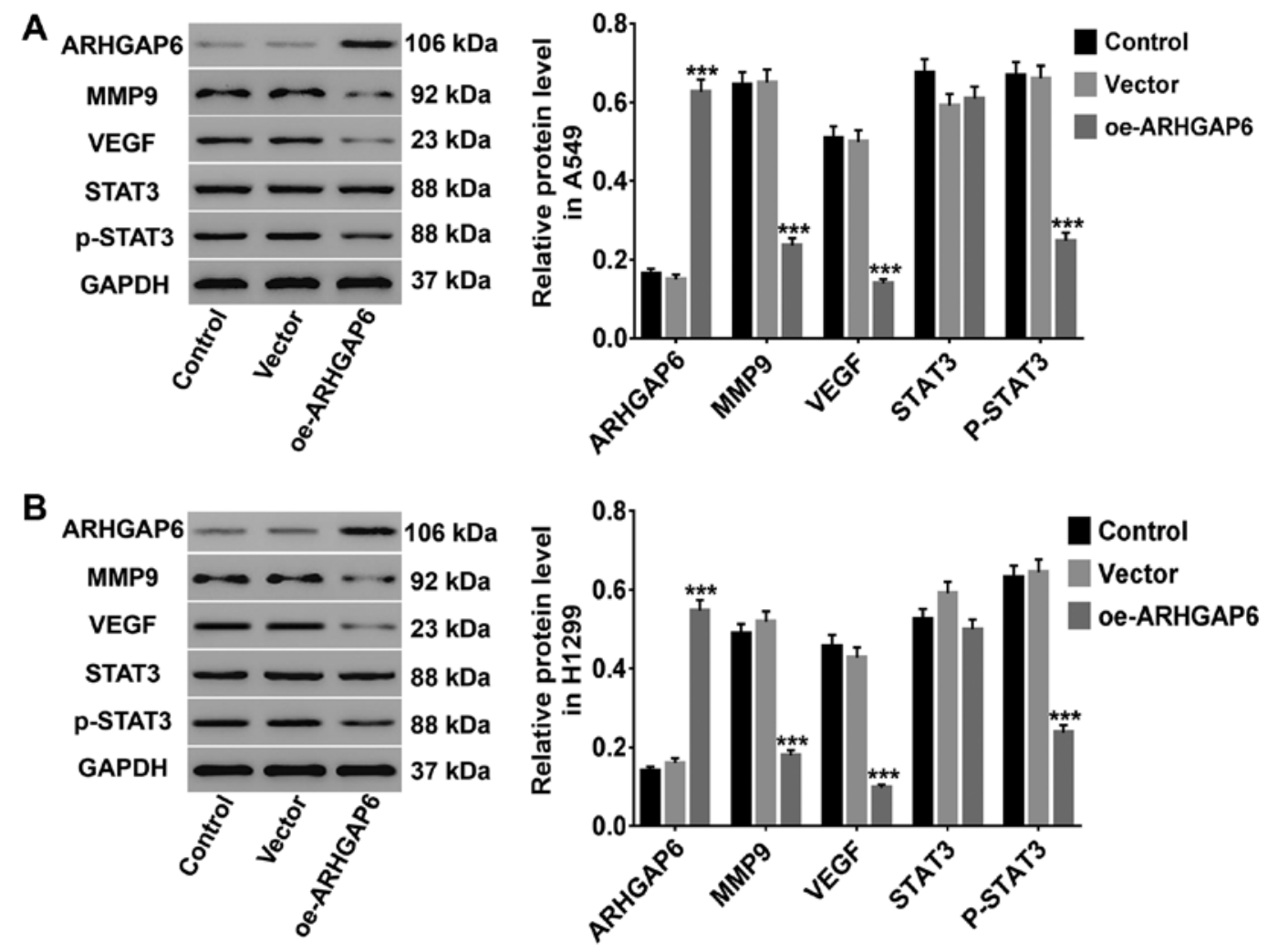

Figure 5. The altered expression of several associated genes after ARHGAP6 upregulation. After ARHGAP6 upregulation, the protein levels of MMP9, VEGF, STAT3 and p-STAT3 in (A) A549 and (B) H1299 cells were detected by western blot analysis. All data are presented as the mean \pm SD of three independent experiments $\left({ }^{* * *} \mathrm{P}<0.001\right.$ compared to the vector). ARHGAP6, Ras homologue GTPase activation protein 6; MMP9, matrix metalloproteinase-9; VEGF, vascular endothelial growth factor.
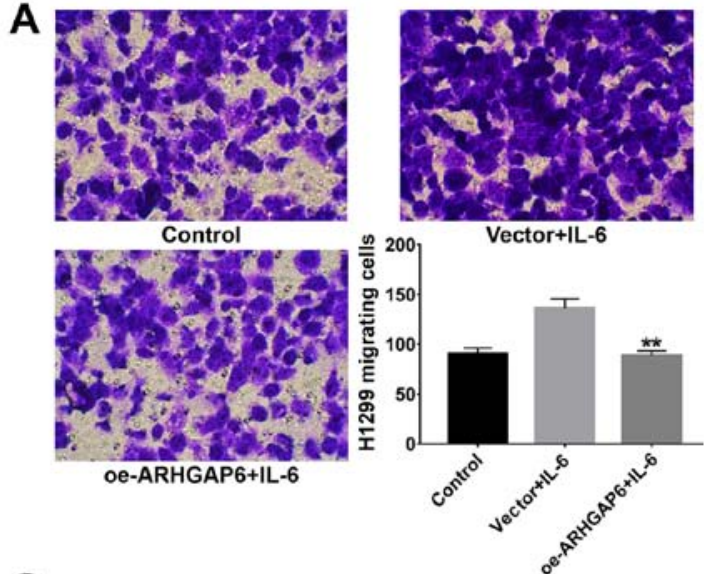
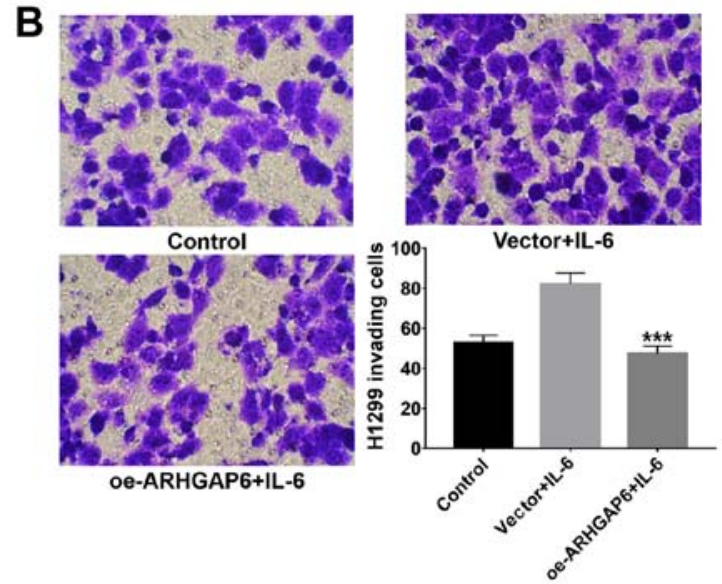

C
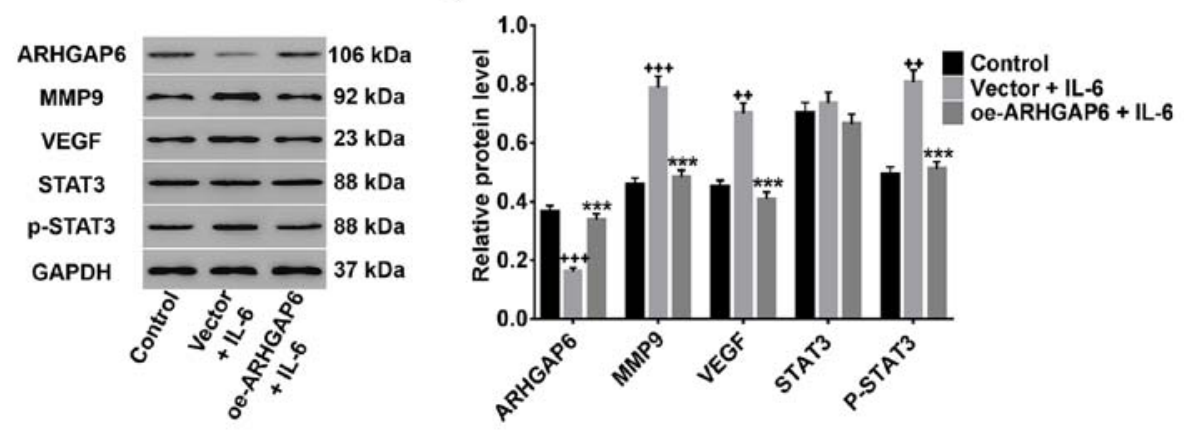

Figure 6. ARHGAP6 upregulation significantly suppresses IL-6-induced migration and invasion, as well as MMP9, VEGF and p-STAT3 expression. After treatment with ARHGAP6 lentivirus and $50 \mathrm{ng} / \mathrm{ml} \mathrm{IL-6,} \mathrm{the} \mathrm{(A)} \mathrm{migrated} \mathrm{and} \mathrm{(B)} \mathrm{invaded} \mathrm{A549} \mathrm{and} \mathrm{H1299} \mathrm{cells} \mathrm{were} \mathrm{determined} \mathrm{by} \mathrm{Transwell} \mathrm{assays.} \mathrm{(C)} \mathrm{In}$ addition, the expression of MMP9, VEGF, STAT3 and p-STAT3 was detected by western blot analysis. All data are presented as the mean \pm SD of three independent experiments $\left({ }^{++} \mathrm{P}<0.01,{ }^{+++} \mathrm{P}<0.001\right.$ compared to the control, ${ }^{* * *} \mathrm{P}<0.001$ compared to the vector + IL-6 group). ARHGAP6, Ras homologue GTPase activation protein 6; MMP9, matrix metalloproteinase-9; VEGF, vascular endothelial growth factor. 


\section{Discussion}

Lung cancer, due to its typical diagnosis at later stages and poor long-term prognosis (35), causes more deaths than other types of cancer, such as breast and colorectal cancer (6). Thus, new potential treatments or prevention methods to reduce the incidence and mortality of lung cancer are urgently needed. Several members of the RhoGAP family have been discovered to function in lung cancer. For example, a study has reported that ARHGAP10 acts as a tumor suppressor in lung cancer (36), and the metastasis of lung cancer cells was effectively suppressed by downregulation of the protumorigenic protein ARHGAP5 (37). In this study, we found that ARHGAP6 upregulation was beneficial for lung cancer treatment in that it suppressed cell proliferation, migration and invasion via inhibition of STAT3 signaling activation and the expression of MMP9 and VEGF.

Multiple studies have observed a significant increase in MMP9 in lung cancer, and MMP9 performs important roles in tumor growth and metastasis $(14,15)$. In addition to lung cancer, other cancers such as breast cancer exhibit high expression of MMP9 (38-40). MMP9 is associated with pathological type, and positive immunostaining for MMP9 has prognostic value for the distant metastasis or local recurrence of lung cancer $(17,41,42)$. VEGF has been reported to induce tumor blood vessel proliferation and possibly contributes to the extravasation of cancer cells and the formation of metastasis (43). These previous findings are consistent with our discovery that ARHGAP6 was decreased and that MMP9 and VEGF were elevated in tumors from lung cancer patients. Similar to ARHGAP10 and ARHGAP5, we concluded that ARHGAP6 is likely a potentially attractive target for lung cancer diagnosis and treatment. ARHGAP6 upregulation had inhibitory effects on the growth and metastasis of A549 and H1299 cells; the high levels of MMP9, VEGF, and p-STAT3 were markedly reduced, while STAT3 was unchanged. These data indicated that ARHGAP6 was critically important for the growth and metastasis of lung cancer cells, and MMP9 and VEGF, which play a previously described crucial role in lung cancer $(18,43)$, were negatively regulated by ARHGAP6 expression. STAT3 signaling, which is activated in NSCLC cells, was reported to regulate VEGF transcription, and disruption of its activity inhibits tumor angiogenesis $(22,23)$. Therefore, we speculated that the inhibitory effects of ARHGAP6 upregulation on the growth and metastasis of lung cancer cells possibly occurs through the suppression of MMP9, VEGF, and STAT3 signaling. This hypothesis was further supported by the finding that ARHGAP6 upregulation significantly suppressed the effects of IL- 6 treatment on lung cancer cells. There are still some limitations in our research such as the small sample size. Although a small sample size may lead to false positives in statistical analysis, our results are still of clinical value. In the future, we will expand the sample size to more fully ascertain our findings.

In conclusion, we found that upregulation of ARHGAP6 had an inhibitory effect on the cell growth and metastasis of lung cancer, possibly through the suppression of MMP9, VEGF, and STAT3 signaling. Targeting ARHGAP6 may be beneficial for the treatment and prevention for lung cancer.

\section{Acknowledgements}

Not applicable.

\section{Funding}

No funding was received.

\section{Availability of data and materials}

All data generated or analyzed during this study are included in this published article.

\section{Authors' contributions}

YW and YM conceived and designed the study. YW, MX, $\mathrm{RH}$ and KX performed the experiments. YW and YM wrote the manuscript. All authors read and approved the manuscript and agree to be accountable for all aspects of the research in ensuring that the accuracy or integrity of any part of the work are appropriately investigated and resolved.

\section{Ethics approval and consent to participate}

All experiments in this study were approved by the Ethics Committee of Liaoning Cancer Hospital and Institute. Written informed consent was obtained from all patients.

\section{Patient consent for publication}

Not applicable.

\section{Competing interests}

The authors declare that they have no competing interests.

\section{References}

1. Bray F, Jemal A, Grey N, Ferlay J and Forman D: Global cancer transitions according to the Human Development Index (2008-2030): A population-based study. Lancet Oncol 13: 790-801, 2012.

2. Ferlay J, Shin HR, Bray F, Mathers C and Parkin DM: GLOBOCAN 2008, cancer incidence and mortality worldwide: IARC CancerBase no. 10. Int J cancer 136: E359-E386, 2012.

3. Jemal A, Bray F, Center MM, Ferlay J, Ward E and Forman D: Global cancer statistics. CA Cancer J Clin 61: 69-90, 2011.

4. Brawley OW, Glynn TJ, Khuri FR, Wender RC and Seffrin JR: The first Surgeon General's report on smoking and health: The 50th anniversary. CA Cancer J Clin 64: 5-8, 2014.

5. Prevention NCfCD, Smoking HPOo and Health: The Health Consequences of Smoking-50 Years of Progress: A Report of the Surgeon General. Public Health Service. Office of the Surgeon General, United States, 2014

6. Nana-Sinkam SP and Powell CA: Molecular biology of lung cancer: Diagnosis and management of lung cancer, 3rd edition: American College of Chest Physicians evidence-based clinical practice guidelines. Chest 143: e30S-e39S, 2013.

7. Siegel R, Naishadham D and Jemal A: Cancer statistics, 2012. CA Cancer J Clin 62: 10-29, 2012.

8. Egile C, Rouiller I, Xu XP, Volkmann N, Li R and Hanein D: Mechanism of filament nucleation and branch stability revealed by the structure of the Arp2/3 complex at actin branch junctions. PLoS Biol 3: e383, 2005.

9. Ridley AJ: Rho-related proteins: Actin cytoskeleton and cell cycle. Curr Opin Genet Dev 5: 24-30, 1995. 
10. SchaeferL,PrakashSandZoghbiHY:Cloning and characterization of a novelrho-type GTPase-activating protein gene (ARHGAPG) from the critical region for microphthalmia with linear skin defects. Genomics 46: 268-277, 1997.

11. Tribioli C, Droetto S, Bione S, Cesareni G, Torrisi MR, Lotti LV, Lanfrancone L, Toniolo D and Pelicci P: An X chromosome-linked gene encoding a protein with characteristics of a rhoGAP predominantly expressed in hematopoietic cells. Proc Natl Acad Sci USA 93: 695-699, 1996.

12. Li J, Yang L and Yin Y: Inhibitory effects of Arhgap6 on cervical carcinoma cells. Tumour Biol 37: 1411-1425, 2016

13. Guo F, Liu Y, Huang J, Li Y, Zhou G, Wang D, Li Y, Wang J and Xie P: Identification of Rho GTPase activating protein 6 isoform 1 variant as a new molecular marker in human colorectal tumors. Pathol Oncol Res 16: 319-326, 2010.

14. Faraji SN, Mojtahedi Z, Ghalamfarsa G and Takhshid MA: $\mathrm{N}$-myc downstream regulated gene 2 overexpression reduces matrix metalloproteinase- 2 and -9 activities and cell invasion of A549 lung cancer cell line in vitro. Iran J Basic Med Sci 18 773-779, 2015

15. Xu X, Cao L, Zhang Y, Yin Y, Hu X and Cui Y: Network analysis of DEGs and verification experiments reveal the notable roles of PTTG1 and MMP9 in lung cancer. Oncol Lett 15: 257-263, 2018.

16. Wójcik E, Jakubowicz J, Skotnicki P, Saskorczyńska B and Kulpa JK: IL-6 and VEGF in small cell lung cancer patients. Anticancer Res 30: 1773-1778, 2010.

17. Cai J, Li R, Xu X, Zhang L, Wu S, Yang T, Fang L, Wu J, Zhu X, Li $M$ and Huang Y: URGCP promotes non-small cell lung cancer invasiveness by activating the NF- $\mathrm{kB}-\mathrm{MMP}-9$ pathway. Oncotarget 6: 36489-36504, 2015.

18. Zuo J, Wen M, Li S, Lv X, Wang L, Ai X and Lei M Overexpression of CXCR4 promotes invasion and migration of non-small cell lung cancer via EGFR and MMP-9. Oncol Lett 14 7513-7521, 2017

19. Goudar RK and Vlahovic G: Hypoxia, angiogenesis, and lung cancer. Curr Oncol Rep 10: 277-282, 2008.

20. Lucchi M, Mussi A, Fontanini G, Faviana P, Ribechini A and Angeletti CA: Small cell lung carcinoma (SCLC): The angiogenic phenomenon. Eur J Cardiothorac Surg 21: 1105, 2002.

21. Yu H and Jove R: The STATs of cancer-new molecular targets come of age. Nat Rev Cancer 4: 94-105, 2004.

22. Leong H, Mathur PS and Greene GL: Green tea catechins inhibit angiogenesis through suppression of STAT3 activation. Breast Cancer Res Treat 117: 505-515, 2009.

23. Song L, Rawal B, Nemeth JA and Haura EB: JAK1 activates STAT3 activity in non-small-cell lung cancer cells and IL-6 neutralizing antibodies can suppress JAK1-STAT3 signaling. Mol Cancer Ther 10: 481-494, 2011.

24. Yeh HH, Lai WW, Chen HH, Liu HS and Su WC: Autocrine IL-6-induced Stat 3 activation contributes to the pathogenesis of lung adenocarcinoma and malignant pleural effusion. Oncogene 25: 4300-4309, 2006

25. Martín F, Santolaria F, Batista N, Milena A, González-Reimers E, Brito MJ and Oramas J: Cytokine levels (IL-6 and IFN-gamma), acute phase response and nutritional status as prognostic factors in lung cancer. Cytokine 11: 80-86, 1999.

26. Yanagawa H, Sone S, Takahashi Y, Haku T, Yano S, Shinohara T and Ogura T: Serum levels of interleukin 6 in patients with lung cancer. Br J Cancer 71: 1095-1098, 1995.

27. Livak KJ and Schmittgen TD: Analysis of relative gene expression data using real-time quantitative PCR and the $2^{-\Delta \Delta C_{\mathrm{T}}}$ method. Methods 25: 402-408, 2001.
28. Hong J, Kang B, Kim A, Hwang S, Ahn J, Lee S, Kim J, Park JH and Cheon DS: Development of a highly sensitive real-time one step RT-PCR combined complementary locked primer technology and conjugated minor groove binder probe. Virol $\mathrm{J} 8$ : $330,2011$.

29. Kobayashi T, Hino S, Oue N, Asahara T, Zollo M, Yasui W and Kikuchi A: Glycogen synthase kinase 3 and h-prune regulate cell migration by modulating focal adhesions. Mol Cell Biol 26: 898-911, 2006.

30. Cheng X, Yang Y, Fan Z, Yu L, Bai H, Zhou B, Wu X, Xu H, Fang M, Shen A, et al: MKL1 potentiates lung cancer cell migration and invasion by epigenetically activating MMP9 transcription. Oncogene 34: 5570-5581, 2015.

31. Takeda A, Stoeltzing O, Ahmad SA, Reinmuth N, Liu W, Parikh A, Fan F, Akagi M and Ellis LM: Role of angiogenesis in the development and growth of liver metastasis. Ann Surg Oncol 9: 610-616, 2002

32. Zhang ZH, Bi-Dan HU, Min YU and Zhang YB: Expressions of leptin, STAT3, p-STAT3 and bcl-2 in lung cancer and their clinical significance. Tumor 30: 529-534, 2010.

33. Heinrich PC,Behrmann I,Haan S,Hermanns HM, Müller-Newen G and Schaper F: Principles of interleukin (IL)-6-type cytokine signaling and its regulation. Biochem J 374: 1-20, 2003.

34. Schafer ZT and Brugge JS: IL-6 involvement in epithelial cancers. J Clin Invest 117: 3660-3663, 2007.

35. Wang T, Nelson RA, Bogardus A and Grannis FW Jr: Five-year lung cancer survival: Which advanced stage nonsmall cell lung cancer patients attain long-term survival? Cancer 116: 1518-1525, 2010.

36. Teng JP, Yang ZY, Zhu YM, Ni D, Zhu ZJ and Li XQ: The roles of ARHGAP10 in the proliferation, migration and invasion of lung cancer cells. Oncol Lett 14: 4613-4618, 2017.

37. Wang J, Tian X, Han R, Zhang X, Wang X, Shen H, Xue L, Liu Y, Yan X, Shen J, et al: Downregulation of miR-486-5p contributes to tumor progression and metastasis by targeting protumorigenic ARHGAP5 in lung cancer. Oncogene 33: 1181-1189, 2014.

38. Zuo J, Ishikawa T, Boutros S, Xiao Z, Humtsoe JO and Kramer RH: Bcl-2 overexpression induces a partial epithelial to mesenchymal transition and promotes squamous carcinoma cell invasion and metastasis. Mol Cancer Re 8: 170-182, 2010.

39. Gao J, Liu X, Yang F, Liu T, Yan Q and Yang X: By inhibiting Ras/Raf/ERK and MMP-9, knockdown of EpCAM inhibits breast cancer cell growth and metastasis. Oncotarget 6: 27187-27198, 2015.

40. Shon SK, Kim A, Kim JY, Kim KI, Yang Y and Lim JS: Bone morphogenetic protein-4 induced by NDRG2 expression inhibits MMP-9 activity in breast cancer cells. Biochem Biophys Res Commun 385: 198-203, 2009.

41. Elbadrawy MK, Yousef AM, Shaalan D and Elsamanoudy AZ: Matrix metalloproteinase-9 expression in lung cancer patients and its relation to serum mmp-9 activity, pathologic type, and prognosis. J Bronchology Interv Pulmonol 21: 327-334, 2014.

42. Liu J, Ping W, Zu Y and Sun W: Correlations of lysyl oxidase with MMP2/MMP9 expression and its prognostic value in non-small cell lung cancer. Int J Clin Exp Pathol 7: 6040-6047, 2014.

43. Matsuyama W, Hashiguchi T, Mizoguchi A, Iwami F, Kawabata M, Arimura K and Osame M: Serum levels of vascular endothelial growth factor dependent on the stage progression of lung cancer. Chest 118: 948-951, 2000. 